\title{
Impedance pH Monitoring: Intra-observer and Inter-observer Agreement and Usefulness of a Rapid Analysis of Symptom Reflux Association
}

\author{
Andrea Tenca, ${ }^{1}$ Pietro Campagnola, ${ }^{2}$ Ivana Bravi, ${ }^{3}$ Luigi Benini, ${ }^{2}$ Daniel Sifrim ${ }^{3}$ and Roberto Penagini ${ }^{1 *}$ \\ ${ }^{1}$ Gastroenterology Unit 2, Università degli Studi di Milano and Fondazione IRCCS “Ca' Granda” - Ospedale Maggiore Policlinico, Milan, Italy; \\ ${ }^{2}$ Gastroenterology Unit, Università degli Studi di Verona and Policlinico GB Rossi, Verona, Italy; and ${ }^{3}$ Barts and The London School of Medicine \\ and Dentistry, Queen Mary University of London, London, UK
}

\section{Background/Aims}

Symptom reflux association analysis is especially helpful for evaluation and management of proton pump inhibitor (PPI) refractory patients. An accurate calculation requires manual editing of 24-hour multichannel intraluminal impedance-pH (MII-pH) tracings after automatic analysis. Intra- and inter-observer agreement as well as reliability of rapid editing confined to the time around symptomatic episodes are unknown. Aim of this study was to explore these topics in a prospective multicenter study.

\section{Methods}

Forty consecutive patients who were off PPI therapy underwent MII-pH recordings. After automatic analysis, their tracings were anonymized and randomized. Three experienced observers, each one trained in a different European center, independently performed manual editing of 24-hour tracings on 2 separate occasions. Values of symptom index and symptom association probability for acid and non acid reflux were transformed into binary response (i.e., positive or negative).

\section{Results}

Intra-observer agreement on symptom reflux association was $92.5 \%$ to $100.0 \%$ for acid and $85.0 \%$ to $97.5 \%$ for non-acid reflux. Inter-observer agreement was $100.0 \%$ for acid and $82.5 \%$ to $95.0 \%$ for non-acid reflux. Values for symptom index and symptom association probability were similar. Concordance between 24-hour and rapid (2 minutes-window before each symptomatic episode) editings for symptom reflux association occured in 39 to 40 patients (acid) and in 37 to 40 (non-acid), depending on the observer.

\section{Conclusions}

Intra- and inter-observer agreement in classifying patients with or without symptom reflux association at manual editing of 24-hour tracings was high, especially for acid reflux. Classifying patients according to a rapid editing showed excellent concordance with the 24-hour one and can be adopted in clinical practice.

\section{(J Neurogastroenterol Motil 2014;20:205-211)}

Key Words

Esophageal pH monitoring; Inter-observer variability; Intra-observer variability

Received: December 20, 2013 Revised: January 25, 2014 Accepted: February 21, 2014

(c) This is an Open Access article distributed under the terms of the Creative Commons Attribution Non-Commercial License (http://creativecommons. org/licenses/by-nc/3.0) which permits unrestricted non-commercial use, distribution, and reproduction in any medium, provided the original work is properly cited.

*Correspondence: Roberto Penagini, MD

Gastroenterology Unit 2, Fondazione IRCCS Ca' Granda - Ospedale Maggiore Policlinico, via Francesco Sforza 35, 20122 Milan, Italy Tel: +39-02-55033595, Fax: +39-02-50320403, E-mail: roberto.penagini@unimi.it

Financial support: This study was supported by a research grant from Sandhill Scientific, Denver, USA (Daniel Sifrim).

Conflicts of interest: Daniel Sifrim receives a research grant from Sandhill Scientific, Denver, USA.

Author contributions: Andrea Tenca, Daniel Sifrim, Roberto Penagini and Luigi Benini planned the study; Andrea Tenca, Pietro Campagnola and Ivana Bravi analyzed the tracings; Andrea Tenca and Roberto Penagini wrote the paper; Daniel Sifrim and Luigi Benini reviewed the paper. All the authors discussed the results. 


\section{Introduction}

Esophageal 24-hour multichannel intraluminal impedance$\mathrm{pH}$ monitoring (MII-pH) is currently considered the gold standard for evaluation of gastro-esophageal reflux disease (GERD). Its advantage over traditional $\mathrm{pH}$-monitoring is the ability to detect weakly acidic reflux episodes in addition to acid reflux and also to differentiate among liquid, gaseous and mixed liquid/gaseous refluxes. ${ }^{1}$ Observations with this technique have shown the clinical relevance of weakly acidic reflux especially in patients poorly responsive to proton pump inhibitors (PPI). ${ }^{2-4}$

Whereas $\mathrm{pH}$-monitoring analysis is automatic and very quick, analysis of MII-pH tracings is much more time consuming because it needs manual revision of tracing after the automatic analysis, especially because events other than reflux are included among reflux episodes by the software. Automatic analysis particularly overestimates the number of non-acid reflux events resulting in a lower sensitivity and specificity of a positive symptom index (SI) compared to visual analysis. ${ }^{5}$ Moreover a low baseline impedance, which may be observed especially in presence of erosive esophagitis or Barrett's esophagus ${ }^{6,7}$ makes the analysis more difficult and the mistakes easier to occur.

Information resulting from MII-pH is important especially in patients refractory to PPIs because it guides medical treatment and may suggest usefulness of anti-reflux surgery. Both a quantitative (i.e., number of reflux episodes) and a qualitative analysis (i.e., symptom reflux association) should be performed, the latter analysis having a higher relevance in PPI refractory patients who frequently have a normal number of reflux episodes. ${ }^{2,3}$ Studies on intra- and inter- observer agreement of manual analysis are scanty, small, referred to the paediatric population or to healthy adults, ${ }^{8-11}$ and they have focused on number of reflux episodes only.

In clinical practice physicians often concentrate their editing in the time window around symptomatic episodes in order to save time, however there are so far no data on reliability of such a partial, quick analysis of MII-pH tracings.

Aims of this study were to evaluate: (1) agreement within and between 3 experienced observers trained in different European Centers for presence/absence of symptom reflux association according to currently used indexes and for detection of individual reflux episodes and (2) concordance between the traditional 24hour manual analysis and a quicker one for presence/absence of symptom reflux association.

\section{Materials and Methods}

\section{Patient Population}

Between September 2011 and January 2012 forty consecutive patients off PPI therapy with typical (i.e., heartburn and regurgitation) and/or atypical (i.e., chest pain) esophageal or extraesophageal (i.e., cough) symptoms possibly related to GERD, who have undergone 24-hour MII-pH in 2 Centers in Northern Italy (Milan and Verona) and have reported symptomatic episodes during the test, were prospectively enrolled. Each center has provided 20 MII-pH tracings. The study protocol has been approved by the Ethics Committees of both hospitals.

\section{Impedance-pH Equipment}

Esophageal MII-pH monitoring was performed using a MII-pH catheter (Z61A; Medical Measurement Systems, Enschede, The Netherlands) containing one distal antimony $\mathrm{pH}$ electrode and eight impedance electrode rings at 2, 4, 6, 8, 10, 14, 16 and $18 \mathrm{~cm}$ from the tip of the catheter. Each pair of adjacent electrodes represents an impedance-measuring segment $(2 \mathrm{~cm}$ in length) corresponding to one recording channel. The eight impedance and $\mathrm{pH}$ signals were recorded at $50 \mathrm{~Hz}$ on a $128 \mathrm{MB}$ Compact Flash Card. Data were stored in a portable receiver with impedance amplifier (Medical Measurement Systems).

\section{Study Protocol}

After an overnight fast, patients attended the Upper Gastrointestinal (GI) Physiology Unit of both Centers. Patient's medical history was collected and informed consent was signed. The lower esophageal sphincter (LES) was located by esophageal manometry and the MII-pH catheter was passed trans-nasally under topical anaesthesia and positioned with the $\mathrm{pH}$ electrode 5 $\mathrm{cm}$ above the upper border of the LES. During a MII-pH monitoring, patients were asked to report timing of meals and periods spent in recumbent position on a daily diary card; when a symptom occurred patients were asked to push a botton on the portable receiver and to report the exact time on the diary card. When many symptoms were reported, only the principal symptom was taken into account. During the recording period patients were allowed to have a free diet, except for known acidic food and beverages, and to continue their usual daily activities. Patients returned to the Upper GI Physiology Unit on the following morning for catheter removal. 
All tracings were read twice by the three observers, each one trained in a different Center (London, Milan and Verona). The three observers were all experienced in the analysis of MII-pH tracings, having previously analyzed at least 200 tracings and performing at least $70 \mathrm{MII}-\mathrm{pH} / \mathrm{year}$. The second reading of each tracing was performed at least 12 weeks after the first one.

\section{Data Analysis}

Data stored on the Compact Flash Card were downloaded into a personal computer. Markers of meal periods and of timing in recumbent position were manually inserted. Data were analysed by using an automated reflux detection algorithm (Medical Measurement Systems) and meal periods were excluded from the analysis. Original tracings were anonimyzed and numbered from 01 to 20 (provided by Milan) and from 41 to 60 (provided by Verona) for inter-observer agreement analysis. These tracings were subsequently duplicated and numbered in a radomized order from 21 to 40 and from 61 to 80 for intra-observer agreement analysis. Each tracing was named adding a code identifying the Center in order to distinguish those reviewed by each observer (observer 1 from Milan, observer 2 from Verona and observer 3 from London). In order to identify tracings difficult to analyse, baseline impedance of each tracing was measured before manual analysis. Baseline esophageal impedance was assessed as a mean baseline at the two most distal impedance channels (situated at 3 and $5 \mathrm{~cm}$ above the LES), considering a 5 -minutes window period during the night. Baseline impedance was considered low if $<$ $500 \Omega .^{7,12}$ The traditional 24-hour manual analysis was performed as follows. Each observer went through every reflux episode and, when he/she did not agree with the automatic analysis, the reflux episode was erased; furthermore reflux episodes not recognized by the automatic analysis were added. In order to avoid a possible bias due to variability of a further analysis, the rapid analysis was obtained by checking for changes that each observer had made during his/her 24-hour analysis in the 2-minute window period preceding each symptom and copying them in a separate automated analysis file.

\section{Definitions}

\section{Reflux episodes}

Only liquid and mixed liquid-gas reflux episodes according to impedance changes were included in the analysis. These reflux episodes were classified by $\mathrm{pH}$ drop nadir in: (1) acid reflux: impedance-detected reflux event with a nadir $\mathrm{pH}$ less than 4, (2) weakly acid reflux: impedance-detected reflux event with a nadir $\mathrm{pH}$ between 4 and 6, and (3) weakly alkaline reflux: impedancedetected reflux event with a nadir $\mathrm{pH}$ above $7 .{ }^{1}$ As weakly alkaline refluxes are very infrequent, in the analysis they were merged with weakly acidic refluxes and considered as non-acid reflux. Total number of reflux episodes was considered pathological when $\geq 75 / 24$ hours. $^{13}$

\section{Symptom-reflux association}

SI and symptom association probability (SAP) were automatically calculated by the software in each patient. Only the association between the principal symptom reported by the patient and acid and non-acid reflux was reported. SI and SAP were defined according to Wiener et $\mathrm{al}^{14}$ and Weusten et $\mathrm{al}^{15}{ }^{15}$ respectively. SI was considered positive when $\geq 50 \%$ and SAP when $\geq 95 \%$.

\section{Statistical Methods}

Intra-observer repeatability and inter-observer reproducibility of esophageal 24-hour pH-impedance analysis were expressed as percentage of agreement and as Cohen's kappa statistic. These measures were applied to agreement on positive/negative symptom-reflux association and on individual reflux episodes. A kappa equal to 0 meant that the agreement was no better than that expected by chance alone, and a kappa values equal to 1 indicated perfect agreement.

Table 1. Variables of the 40 Multichannel Intraluminal 24-hour Impedance-pH Tracings as Assessed by the 3 Observers

\begin{tabular}{lccc}
\hline & $\begin{array}{c}\text { Observer 1 } \\
\text { (Milan) }\end{array}$ & $\begin{array}{c}\text { Observer 2 } \\
\text { (Verona) }\end{array}$ & $\begin{array}{c}\text { Observer 3 } \\
\text { (London) }\end{array}$ \\
\hline $\begin{array}{c}\text { AC reflux episodes } \\
\text { (median [range] })\end{array}$ & $25(1-90)$ & $22(0-83)$ & $25(1-91)$ \\
$\begin{array}{c}\text { NA reflux episodes } \\
\text { (median [range]) }\end{array}$ & $19(2-89)$ & $8(1-76)$ & $21(2-99)$ \\
$\begin{array}{l}\text { Total reflux episodes } \\
(\text { median [range]) }\end{array}$ & $44(5-99)$ & $28(1-89)$ & $49(4-106)$ \\
$\begin{array}{l}\text { Positive SI for AC (n [\%]) } \\
\text { Positive SI for NA (n [\%]) }\end{array}$ & $5(12.5)$ & $5(12.5)$ & $5(12.5)$ \\
$\begin{array}{l}\text { Positive SAP for AC (n [\%]) } \\
\text { Positive SAP for NA (n [\%]) }\end{array}$ & $6(15.0)$ & $0(0.0)$ & $1(2.5)$ \\
\hline
\end{tabular}

AC, acid; NA, non acid; SI, symptom index; SAP, symptom association probability. 
Table 2. Kappa Values With Standard Error Between the First and the Second Analysis, With Regards to Symptom Index and Symptom Association Probability Divided Into Acid/Non acid

\begin{tabular}{lccccc}
\hline & \multicolumn{2}{c}{ SI } & & \multicolumn{2}{c}{ SAP } \\
\cline { 2 - 3 } \cline { 5 - 6 } & Acid & Non acid & & Acid & Non acid \\
\hline Observer 1 & $0.77(0.15)$ & $0.65(0.31)$ & & $0.80(0.13)$ & $0.48(0.18)$ \\
Observer 2 & $0.77(0.15)$ & ${ }^{a}$ & & $0.72(0.15)$ & $0.79(0.20)$ \\
Observer 3 & $1.00(0.00)$ & ${ }^{-}$ & & $1.00(0.00)$ & $0.54(0.20)$ \\
\hline
\end{tabular}

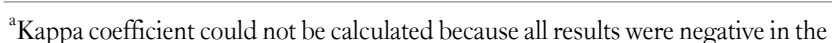
second analysis.

SI, symptom index; SAP, symptom association probability.

Data are presented as kappa coefficient (standard error).

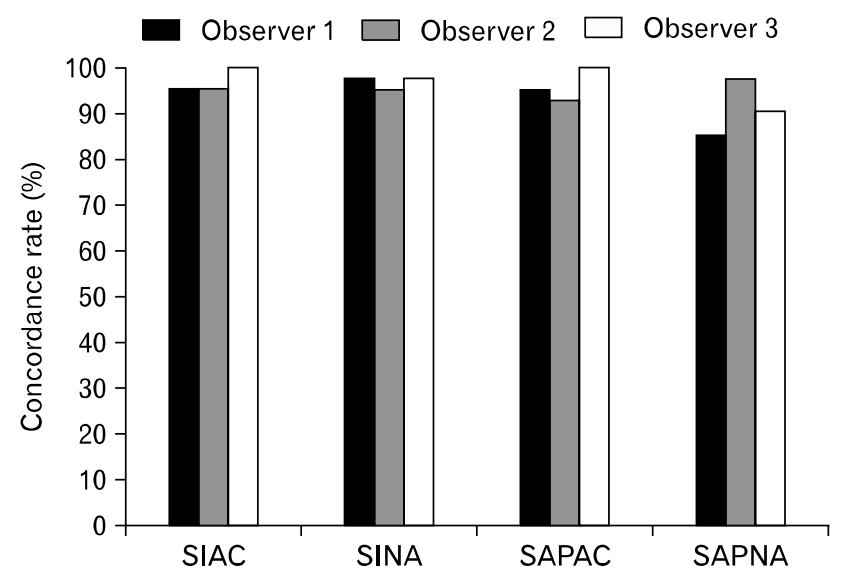

Figure 1. Percentage of concordance between the first and the second analysis, with regards to symptom index (SI) and symptom association probability (SAP) divided into acid (AC) and non-acid (NA) reflux.

\section{Results}

\section{Patients Characteristics}

Twenty-three of the 40 enrolled patients were women and the median age was 55 years (range, 27 to 88 years). All patients completed the study and the recording period was more than 23 hours in all of them. Seventeen (42.5\%), 7 (17.5\%) and 16 patients $(40 \%)$ experienced typical symptoms, chest pain and cough, respectively. All patients had a previous upper GI endoscopy showing grade A erosive esophagitis in 5/40 patients (12.5\%). No Barrett's esophagus was detected. No patients had a low esophageal impedance baseline with the median value being $2487 \Omega$ (range, 662-5548 $\Omega$ ). Table 1 shows variables of the 40 MII-pH tracings as assessed by the 3 observers. The total num-
Table 3. Kappa Values With Standard Error Between Observers, With Regards to Symptom Index and Symptom Association Probability Divided Into Acid/Non acid

\begin{tabular}{|c|c|c|c|c|}
\hline & \multicolumn{2}{|c|}{ SI } & \multicolumn{2}{|c|}{ SAP } \\
\hline & Acid & Non acid & Acid & Non acid \\
\hline $\begin{array}{c}\text { Observer } 1 \\
\text { vs. }\end{array}$ & $1.00(0.00)$ & $-{ }^{\mathrm{a}}$ & $1.00(0.00)$ & $0.35(0.19)$ \\
\hline Observer 2 & & & & \\
\hline $\begin{array}{c}\text { Observer } 1 \\
\text { vs. }\end{array}$ & $1.00(0.00)$ & $-{ }^{a}$ & $1.00(0.00)$ & $0.36(0.19)$ \\
\hline $\begin{array}{c}\text { Observer } 2 \\
\text { vs. } \\
\text { Observer } 3\end{array}$ & $1.00(0.00)$ & $-{ }^{a}$ & $1.00(0.00)$ & $0.23(0.23)$ \\
\hline
\end{tabular}

${ }^{a}$ Kappa coefficient could not be calculated because all results were negative for observer 1 and 2 .

SI, symptom index; SAP, symptom association probability.

Data are presented as kappa coefficient (standard error).

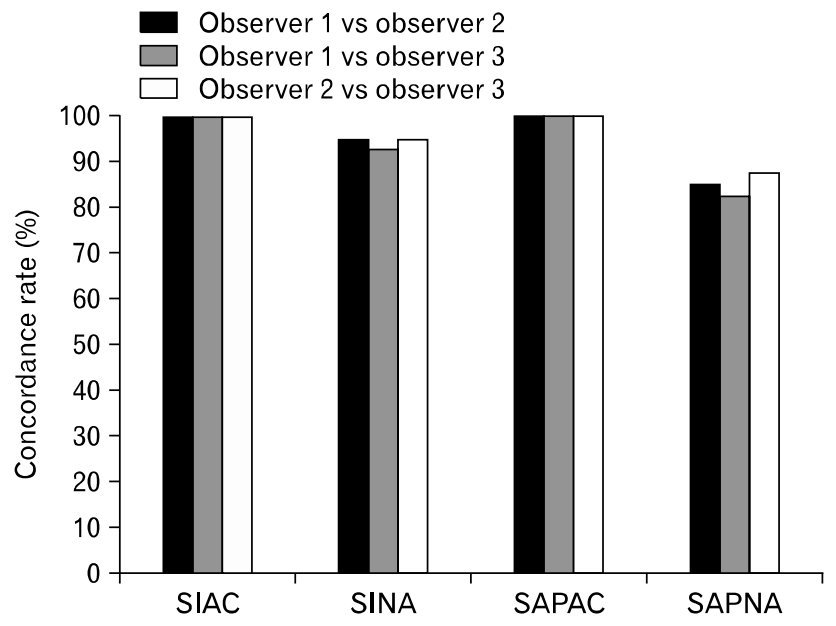

Figure 2. Percentage of concordance between observers, with regards to symptom index (SI) and symptom association probability (SAP) divided into acid (AC) and non-acid (NA) reflux.

ber of reflux episodes was more than 75 in $5 / 40$ (12.5\%), 2/40 $(5 \%)$ and $5 / 40(12.5 \%)$ tracings for observer 1,2 and 3 , respectively.

\section{Agreement for Symptom/Reflux Association}

\section{Intra-observer (see Table 2 and Fig. 1)}

Agreement between the first and the second analysis was generally high for all 3 observers and slightly better for acid compared to non-acid refluxes. 


\section{Inter-observer (see Table 3 and Fig. 2).}

Agreement between the 3 observers was generally good, although it was higher for acid refluxes compared to non-acid ones.

\section{Agreement for Detection of Individual Reflux Episodes}

\section{Intra-observer}

Median intra-observer agreement between first and second analysis was $98.2 \%$ (range, 92.4-99.6\%) for acid episodes with a median kappa coefficient of 0.68 whereas it was $92.3 \%$ (range, 82.1-92.4\%) for non-acid episodes with a median kappa coefficient of 0.40. Intra-observer agreement for judging a study normal or pathological on the basis of the number of reflux episodes was almost perfect for all the observers, as the number of studies with a pathological number of reflux episodes remained the same for observer 2 and increased from 5 to $6 / 40$ for observer 1 and 3 in the second analysis.

\section{Inter-observer}

Median inter-observer agreement was $86.8 \%$ (range, 86.3$97.6 \%$ ) for acid episodes with a median kappa coefficient of 0.22 . Median agreement was lower for non-acid episodes, $55.7 \%$ (range, 48.9-81.5\%) with a median kappa of 0.19 .

\section{Symptom Reflux Association: Concordance Between 24-hour and a Rapid Analysis}

Rapid editing showed to be highly predictive of the traditional 24-hour one for all 3 observers with regards to the four symptom reflux association variables (Table 4 ). In particular rapid and traditional 24-hour editing showed concordance in 39 (97.5\%) to 40

Table 4. Percentage of Concordance With Confidence Interval Between Short and Traditional Analysis

\begin{tabular}{cccccc}
\hline & \multicolumn{2}{c}{ SI } & & \multicolumn{2}{c}{ SAP } \\
\cline { 2 - 3 } \cline { 5 - 6 } & Acid & Non-acid & & Acid & Non-acid \\
\hline Observer 1 & 100.0 & 100.0 & & 100.0 & 97.5 \\
& $(91-100)$ & $(91-100)$ & & $(91-100)$ & $(85-100)$ \\
Observer 2 & 97.5 & 95.0 & & 97.5 & 92.5 \\
& $(85-100)$ & $(82-99)$ & & $(85-100)$ & $(78-98)$ \\
Observer 3 & 97.5 & 100.0 & & 100.0 & 100.0 \\
& $(85-100)$ & $(91-100)$ & & $(91-100)$ & $(91-100)$ \\
\hline
\end{tabular}

SI, symptom index; SAP, symptom association probability. Data are presented as percentage of concordance (confidence interval).
(100.0\%) patients for acid reflux and in 37 (92.5\%) to 40 (100.0\%) for non-acid reflux, depending on the observer.

\section{Discussion}

Results of our study showed that intra- and inter-observer agreement for presence or absence of a symptom-reflux association was high, though slightly lower for non acid (82.0-97.5\%) than for acid reflux (92.0-100.0\%). Furthermore, and more interestingly from a practical point of view, a rapid analysis of symptom reflux association confined to the time around symptomatic episodes was highly predictive of the analysis performed over 24 hours.

Evaluation of symptom/reflux association is the most useful variable in the analysis of MII-pH monitoring performed in patients referred to specialized Centers, who are frequently PPI resistant and often with normal reflux exposure. ${ }^{2,3,16}$ When symptom reflux association is negative both for acid and non-acid reflux in a patient with normal reflux the diagnosis of GERD is ruled out and anti-reflux surgery is no longer indicated as a therapeutic option. Automatic analysis by computer software has a rather low reliability for classifying patients as having positive or negative symptom reflux association because it overestimates reflux episodes ${ }^{5,8,10}$ and especially weakly acid reflux, ${ }^{5}$ when compared to manual analysis. This is why tracings are manually edited by a physician after automatic analysis, a routine which opens to possible inaccuracies due to intra and inter-observer variability, which have never been evaluated.

Our study is the first one which has focused on this topic and has shown good agreement both within each observer and among observers. Results of kappa statistics were less satisfactory especially regarding non-acid reflux because the vast majority of patients had a negative symptom reflux association and it is known that the imbalance between the 2 options of a binary response weakens this statistical analysis. Furthermore, kappa was not calculated for non acid reflux on 5 occasions, because all patients had a negative symptom reflux association. Our series is similar to previous ones, where esophageal symptoms were frequently found to be functional. ${ }^{17}$

Our study investigated also intra- and inter-observer agreements on number of acid and non-acid reflux episodes. Previous studies have addressed this topic in the paediatric ${ }^{8,9}$ and in the adult healthy ${ }^{11}$ and GERD population, ${ }^{10}$ although the 2 latter studies looked at inter-observer agreement only. Furthermore in the paper by Ravi et $\mathrm{al}^{10}$ analysis was limited to classifying trac- 
ings with either normal or pathological number of reflux episodes. In the report by Loots et $\mathrm{al}^{8} 10$ tracings were analysed and inter-observer agreement was calculated among 10 assessors whereas intra-observer agreement was measured among 3 of them. In the report by Pilic et $\mathrm{al}^{9} 24$ tracings were analysed for inter-observer agreement and 6 for intra-observer agreement between 2 investigators. In the study by Zerbib et $\mathrm{al}^{11} 20$ tracings off PPIs and 18 on PPIs obtained from 20 healthy subjects were evaluated for agreement between 2 observers. It is not easy to compare previous reports with ours because those studies considered all reflux episodes together, i.e., without separating acid and non-acid reflux, and data were presented in different formats. Regarding inter-observer agreement, the 2 observers in the study by Pilic et al ${ }^{9}$ had an agreement which varied widely among the 24 tracings, from 0 to $98 \%$ with a median of $73 \%$, whereas the 2 observers in the study by Zerbib et al ${ }^{11}$ had an overall agreement of $84 \%$ off PPIs and $73 \%$ on PPIs. Our results, obtained analyzing 40 tracings, showed lower inter-observer agreement on number of non-acid compared to acid reflux episodes (48.9-81.5\% vs. $86.3-97.6 \%$ ). This result was presumably mainly related to observer 2 scoring a lower number of non-acid refluxes, a fact which was likely to have also contributed to a lower inter-observer agreement on symptom reflux association for non-acid in comparison with acid reflux. Episodes of non-acid reflux are thus more challenging to be agreed upon both between software and an experienced assessor ${ }^{5}$ and among experienced assessors. Reasons for this have not been explored in detail, however absence of a clear drop in $\mathrm{pH}$ in situations where impedance readings are more difficult to be interpreted, i.e., unclear flow direction, flow after a swallow, possible reflux during low baseline impedance and patterns containing gas, presumably are important variables. ${ }^{18}$ These observations should stimulate on one hand the manufacturers to improve the software used for automatic analysis and on the other the training Centers to more carefully train physicians approaching this clinical field. Consensus meetings among experts with the aim to propose patterns to be detected as gastroesophageal reflux in MII-pH tracing are welcome in order to improve both automatic analysis and inter-observer agreement. ${ }^{18}$ A lower agreement for number of reflux episodes has been suggested in technically challenging tracings in children and infants. ${ }^{8}$ We have measured baseline impedance in order to classify tracings of technical difficulty, although others have suggested additional variables, ${ }^{18}$ and found that none was challenging because baseline impedance was $>500 \Omega$ in all tracings. Our finding is in agreement with previous data, ${ }^{6,19}$ the vast majority of our patients being endoscopy negative and with low acid exposure.

A drawback of manual editing after automatic analysis is that reviewing the whole tracing is time consuming and a quicker analysis confined to the time around symptomatic episodes has been advocated in clinical practice. This is why we think that the clinically more relevant information coming from our paper is that presence/absence of symptom reflux association determined after a rapid analysis of MII-pH recordings, i.e., centered in a 2-minute time window before symptomatic episodes, was highly predictive of results after the complete 24-hour analysis. This was true both for acid and non-acid reflux. Our observation thus strongly suggests reliability of a quick manual editing, which would save physician's time and Health Care System resources.

In conclusion, results of our prospective multicenter study have shown good intra-observer and inter-observer agreement for positive/negative symptom reflux association, when MII-pH tracings are manually edited after automatic analysis. This related both to acid and non-acid reflux, although inter-observer agreement was lower for non-acid reflux. Furthermore they have produced evidence that a rapid manual editing of the automatic analysis confined to a short time window before each symptomatic episode is highly reliable and can be adopted in routine clinical practice.

\section{Acknowledgements}

We thank Dr Ivan Cortinovis of Department of Clinical Science and Community Health, Università degli Studi of Milan, Italy for statistical analysis.

\section{References}

1. Sifrim D, Castell D, Dent J, Kahrilas PJ. Gastro-oesophageal reflux monitoring: review and consensus report on detection and definitions of acid, non-acid, and gas reflux. Gut 2004;53:1024-1031.

2. Zerbib F, Roman S, Ropert A, et al. Esophageal pH-impedance monitoring and symptom analysis in GERD: a study in patients off and on therapy. Am J Gastroenterol 2006;101:1956-1963.

3. Mainie I, Tutuian R, Shay S, et al. Acid and non-acid reflux in patients with persistent symptoms despite acid suppressive therapy: a multicenter study using combined ambulatory impedance- $\mathrm{pH}$ monitoring. Gut 2006;55:1398-1402.

4. Bredenoord AJ, Weusten BL, Timmer R, Conchillo JM, Smout AJ. Addition of esophageal impedance monitoring to $\mathrm{pH}$ monitoring increases the yield of symptom association analysis in patients off PPI therapy. Am J Gastroenterol 2006;101:453-459.

5. Roman S, Bruley des Varannes S, Pouderoux P, et al. Ambulatory 24-h oesophageal impedance-pH recordings: reliability of automatic 
analysis for gastro-oesophageal reflux assessment. Neurogastroenterol Motil 2006;18:978-986.

6. Farré R, Blondeau K, Clement D, et al. Evaluation of oesophageal mucosa integrity by the intraluminal impedance technique. Gut 2011;60:885-892.

7. Hemmink GJ, Alvarez Herrero L, Bogte A, et al. Esophageal motility and impedance characteristics in patients with Barrett's esophagus before and after radiofrequency ablation. Eur J Gastroenterol Hepatol 2013;25:1024-1032.

8. Loots CM, van Wijk MP, Blondeau K, et al. Interobserver and intraobserver variability in $\mathrm{pH}$-impedance analysis between 10 experts and automated analysis. J Pediatr 2012;160:441-446, e1.

9. Pilic D, Höfs C, Weitmann S, et al. Inter- and intraobserver agreement in 24-hour combined multiple intraluminal impedance and $\mathrm{pH}$ measurement in children. J Pediatr Gastroenterol Nutr 2011;53: 255-259.

10. Ravi K, DeVault KR, Murray JA, Bouras EP, Francis DL. Inter-observer agreement for multichannel intraluminal impedance-pH testing. Dis Esophagus 2010;23:540-544.

11. Zerbib F, Roman S, Bruley Des Varannes S, et al. Normal values of pharyngeal and esophageal 24-hour $\mathrm{pH}$ impedance in individuals on and off therapy and interobserver reproducibility. Clin Gastroenterol Hepatol 2013;11:366-372.

12. Heard R, Castell J, Castell DO, Pohl D. Characterization of patients with low baseline impedance on multichannel intraluminal impedance-
pH reflux testing. J Clin Gastroenterol 2012;46:e55-e57.

13. Zerbib F, des Varannes SB, Roman S, et al. Normal values and day-to-day variability of 24-h ambulatory oesophageal impedance- $\mathrm{pH}$ monitoring in a Belgian-French cohort of healthy subjects. Aliment Pharmacol Ther 2005;22:1011-1021.

14. Wiener GJ, Richter JE, Copper JB, Wu WC, Castell DO. The symptom index: a clinically important parameter of ambulatory 24-hour esophageal pH monitoring. Am J Gastroenterol 1988;83:358-361.

15. Weusten BL, Roelofs JM, Akkermans LM, Van Berge-Henegouwen GP, Smout AJ. The symptom-association probability: an improved method for symptom analysis of 24-hour esophageal $\mathrm{pH}$ data. Gastroenterology 1994;107:1741-1745.

16. Savarino E, Zentilin $\mathrm{P}$, Tutuian $\mathrm{R}$, et al. The role of nonacid reflux in NERD: lessons learned from impedance-pH monitoring in $150 \mathrm{pa}-$ tients off therapy. Am J Gastroenterol 2008;103:2685-2693.

17. Savarino E, Pohl D, Zentilin P, et al. Functional heartburn has more in common with functional dyspepsia than with non-erosive reflux disease. Gut 2009;58:1185-1191.

18. Loots CM, Van Wijk M, Omari T, et al. Consensus meeting report: what patterns should be detected as gastroesophageal reflux in pH-impedance recordings? Gut 2012;61(suppl 3):A197.

19. Kessing BF, Bredenoord AJ, Weijenborg PW, Hemmink GJ, Loots CM, Smout AJ. Esophageal acid exposure decreases intraluminal baseline impedance levels. Am J Gastroenterol 2011;106:2093-2097. 